\title{
THE ANALYSIS AND CALCULATION OF ELECTROMAGNETIC FIELD AROUND OVERHEAD POWER LINE
}

\author{
HongWang Yang \\ Department of electrical engineering , North China Electric Power University, Baoding 071000, China \\ 956598589@qq.com
}

Keywords: electromagnetic field, Power System Analysis, Biot-Savart Law

\begin{abstract}
Distribution network is the connection of the generate system and transmission line, which is at the last stage of the process of the energy transportation. It is complex to judge the direction of the power flow, so that it is significant to study a more effective method to judge the direction of the power flow in the Overhead power line.

This paper introduces the principle of the Biot-Savart Law firstly and then introduces the necessity of power system simulation,. To complete the analysis and calculation of electromagnetic field around Overhead power line when Signals Phase Earth Fault occur in the power system, the spatial rectangular coordinate system was established based on the arrangement of the Overhead power line. After the study of $10 \mathrm{KV}$ distribution network we create fault simulation platform ATPDraw and based on MATLAB simulation software, we use the electromagnetic transient program graphical input function and the strong calculation function of MATLAB to calculate electromagnetic field around Overhead power line, so as to provide the basis for further analysis.
\end{abstract}

\section{Introduction}

The Magnetic induction $\mathrm{dB}$, which produced by a current element Idl at a point $\mathrm{P}$ in space, is proportional to the value of the current element $I d l$ and the sine of the angle between the position vector that pointing the current element to the point $\mathrm{P}$ with the vector of current element Idl , and inversely to the distance between $I d l$ and point $\mathrm{P}$.

In former formula, $\frac{u_{0}}{4 \pi}$ is the ratio coefficient, $u_{0}$ is vacuum permeability, whose value is $4 \pi \times 10^{-7} T^{*} \mathrm{~m} / \mathrm{A}$ and the direction is perpendicular to the surface determined by the current element and vector $r$, curve the finger and the direction of thumb is the direction of $\mathrm{dB}$. That is $I d l, \mathrm{~dB}$ and $r$ accord the right-hand screw rule.

The law of Biot Savart is proved by the H.C. Oster experiment. The experiment illustrate that the force which is given by long straight current-carrying to the magnetic pole is horizontal force. J.B. Biot and F.Savart believe that the force that current element gives to magnetic pole is perpendicular to the surface determined by current element and the magnetic pole, in a same way, a horizontal force. They make a conclusion about the relationship between the force and the angle of the bucking current-carrying,as well as the relationship with distance via the experiment that measure the force given by straight and curve current-carrying to the magnetic sole. With the help of P.S.M. Laplace, they got the rule of the effect of the current element to the magnetic pole. According to the view of the close range function ,the conclusion is understand by public as a rule of the production of magnetic field .

\section{Model}

\subsection{Model assumptions}

1) Assume that the Three-phase Conducting Wires of Overhead Transmission Line is in the in the triangle array. 


\begin{tabular}{|l|}
\hline Symbols Definitions \\
\hline$\left(X_{k}, Y_{k}\right)$ the coordinate of the Three-phase Conducting Wires of Overhead Transmission Line \\
$S_{a}$ the coefficient of horizontal magnetic field \\
$W_{0} \quad$ the vertical magnetic field coefficient \\
$(x, y)$ the locations of the sensors \\
$i_{0} \quad$ the zero sequence current \\
$i_{1} \quad$ Positive sequence current \\
$i_{2} \quad$ Negative sequence current
\end{tabular}

2) The weather is normal.

3) There is no other magnetic field and electric source in the field.

\subsection{Symbols and definitions}

\section{3 the model of magnetic field}

According to technical specification for design of overhead distribution lines,power distribution line mainly adopts triangle arrangement or horizontal alignment.A three-phase conductor is infinitely long and is parallel to the ground, The instantaneous current in the three phase wire is Ia, Ib, Ic.We set up a coordinate system in a plane perpendicular to the three-phase wire as shown in the figure below. Three phase conductor coordinates is $(X k, Y k), k=a, b, c$, the coordinates of the testing point $\mathrm{P}$ is $(\mathrm{x}, \mathrm{y})$.

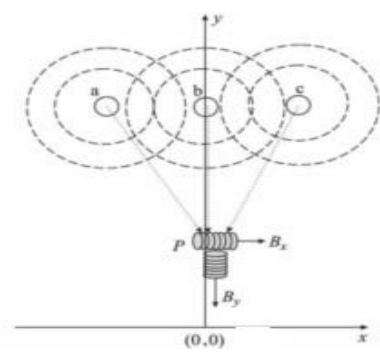

Figure 1 Power line around the magnetic field calculation

The horizontal component of the magnetic induction intensity of $\mathrm{P}$ can be represented as: $B_{x}=S_{a} i_{a}+S_{b} i_{b}+S_{c} i_{c}$

where

$$
\begin{aligned}
& S_{a}=\frac{\mu_{0}}{2 \pi} \frac{Y_{a}-y}{\left(X_{a}-x\right)^{2}+\left(Y_{a}-y\right)^{2}} \\
& S_{b}=\frac{\mu_{0}}{2 \pi} \frac{Y_{b}-y}{\left(X_{b}-x\right)^{2}+\left(Y_{b}-y\right)^{2}} \\
& S_{c}=\frac{\mu_{0}}{2 \pi} \frac{Y_{c}-y}{\left(X_{c}-x\right)^{2}+\left(Y_{c}-y\right)^{2}}
\end{aligned}
$$

Among them Sa,Sb,Sc is the coefficient of horizontal magnetic field, u0 is the air permeability. The vertical component of the magnetic induction intensity of $\mathrm{P}$ is:

$$
B_{y}=W_{a} i_{a}+W_{b} i_{b}+W_{c} i_{c}
$$

Among them $\mathrm{Wa}, \mathrm{Wb}, \mathrm{Wc}$ is the coefficient of vertical magnetic field , $\mathrm{u} 0$ is the air permeability.

When the constraint conditions are met, the coefficient of vertical magnetic field can be described:

$$
\left\{\begin{array}{l}
W_{a}=\frac{\mu_{0}}{2 \pi} \frac{X_{a}-x}{D^{2}} \\
W_{b}=\frac{\mu_{0}}{2 \pi} \frac{X_{b}-x}{D^{2}} \\
W_{c}=\frac{\mu_{0}}{2 \pi} \frac{X_{c}-x}{D^{2}}
\end{array}\right.
$$

According to Karrenbauer Transform, vertical magnetic field is represented as followers:

$$
B_{y}=W_{0} i_{0}+W_{1} i_{1}+W_{2} i_{2}
$$

Where: $W_{0}=W_{a}+W_{b}+W_{c} ; W_{1}=W_{a}-2 W_{b}+W_{c} ; W_{2}=W_{a}+W_{b}-2 W_{c}$ 
As is shown in the Figure 1, Three-phase line is symmetry about tower and three-phase circuit meet the following relationship:

$$
\left\{\begin{array}{l}
X_{c}=-X_{a}=d \\
X_{b}=0
\end{array}\right.
$$

Where $\mathrm{d}$ is a horizon distance between two-phase circuit in outside and the tower. The coefficients can be simplified as:

The vertical magnetic field is:

$$
\begin{gathered}
W_{0}=-\frac{3 \mu_{0}}{2 \pi} \frac{x}{D^{2}} \\
W_{1}=\mathbf{O} \\
W_{2}=-\frac{3 \mu_{0}}{2 \pi} \frac{d}{D^{2}}
\end{gathered}
$$

$$
B_{y}=-\frac{3 \mu_{0}}{2 \pi} \frac{1}{D^{2}}\left(x i_{0}+d i_{2}\right)
$$

Specially, When the nearby checkpoint $\mathrm{P}$ located just below the line, in other words, when $d>>|x| \approx 0$, the vertical magnetic field is:

$$
B_{y}=-\frac{3 \mu_{0}}{2 \pi} \frac{d}{D^{2}} i_{2}
$$

That line just below the vertical filed is proportional to the current and negative sequence.

When the checkpoint is met,the vertical magnetic field is:

$$
B_{y}=-\frac{3 \mu_{0}}{2 \pi} \frac{x}{D^{2}} i_{0}
$$

That is, when the point is far away from the three-phase line in the horizontal direction, vertical magnetic filed proportional to the zero sequence current.

\section{The analysis of electric field}

Using equivalent charge, we can calculate the electric filed strength around the three-phase overhead lines, which is shown as figure.

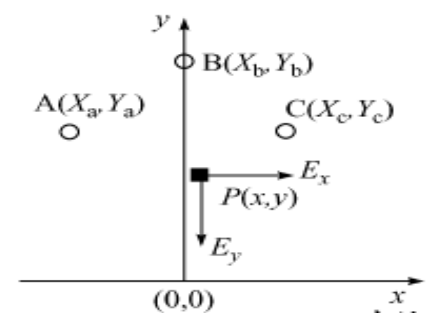

Figure 2 electric filed coordinate system

Assuming the three phase conductor disposed coordinates is $\left(X_{k}, Y_{k}\right), k=a, b, c$, detecting coordinates of point $\mathrm{P}$ is $(x, y)$.Provided that ground potential is 0 and $\mathrm{A}, \mathrm{B}, \mathrm{C}$, for the three phase conductors of potential respectively is $u_{a}, u_{b}, u_{c}$; Unit length of the line were the equivalent charge is $\tau_{a}, \tau_{b}, \tau_{c}$. We can get that:

$$
\left[\begin{array}{c}
\tau_{\mathrm{a}} \\
\tau_{b} \\
\tau_{c}
\end{array}\right]=\left[\begin{array}{lll}
\lambda_{11} & \lambda_{12} & \lambda_{13} \\
\lambda_{21} & \lambda_{22} & \lambda_{23} \\
\lambda_{31} & \lambda_{32} & \lambda_{33}
\end{array}\right]^{-1}\left[\begin{array}{l}
u_{a} \\
u_{b} \\
u_{c}
\end{array}\right]=\lambda^{-1}\left[\begin{array}{l}
u_{a} \\
u_{b} \\
u_{c}
\end{array}\right]
$$

Where $\lambda$ is self potential coefficient of each wire and potential coefficient matrix, which can be obtained according to the method of images.Vertical electric filed $E_{y}$ detected by the three-phase voltage at the common point $\mathrm{P}$ generated and Horizontal electric filed $\mathrm{E}_{x}$ are defined as follows:

$$
\begin{aligned}
& E_{y}=\left[L_{a}, L_{b}, L_{c}\right]\left[\tau_{a}, \tau_{b}, \tau_{c}\right]^{T} \\
& E_{\mathrm{x}}=\left[H_{a}, H_{b}, H_{c}\right]\left[\tau_{a}, \tau_{b}, \tau_{c}\right]^{T}
\end{aligned}
$$


Where

$$
L_{\mathrm{k}}=\frac{1}{2 \pi \varepsilon_{0}}\left[\frac{y-Y_{k}}{\left(x-X_{k}\right)^{2}+\left(y-Y_{k}\right)^{2}}-\frac{y+Y_{k}}{\left(x-X_{k}\right)^{2}+\left(y+Y_{k}\right)^{2}}\right]
$$

\section{Conclusion}

$$
H_{k}=\frac{1}{2 \pi \varepsilon_{0}}\left[\frac{x-X_{k}}{\left(x-X_{k}\right)^{2}+\left(y-Y_{k}\right)^{2}}-\frac{x+X_{k}}{\left(x-X_{k}\right)^{2}+\left(y+Y_{k}\right)^{2}}\right]
$$

After run in the ATP-EMTP we can obtain the Current image:
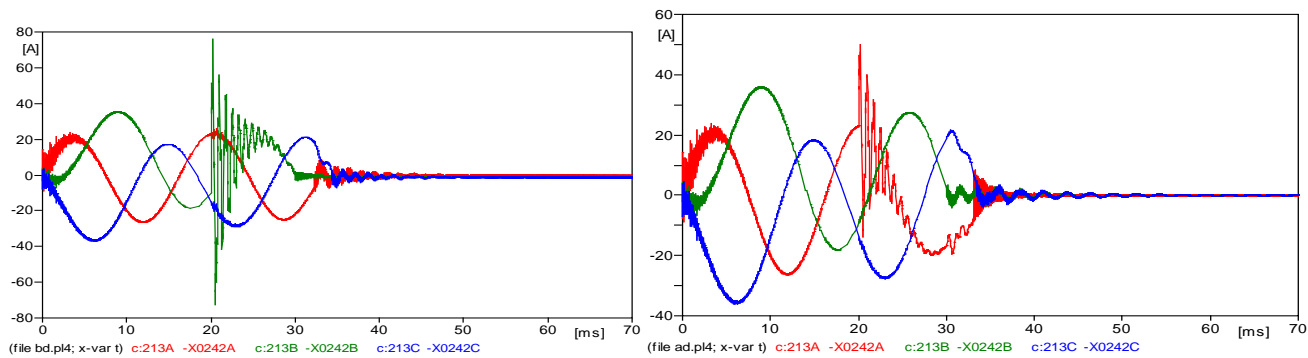

Figure 3 the Current image

To calculate the electromagnetic,we program in MATLAB.it is run as flows:
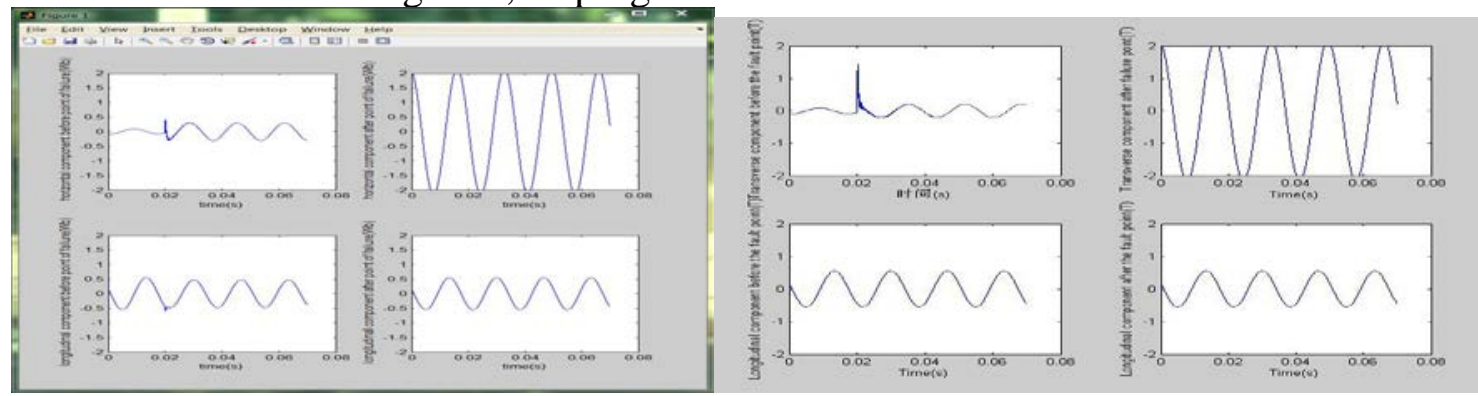

Figure 4 electromagnetic image

Distribution network is the connection of the generate system and current-carrying, which is at the last stage of the process of the energy transportation. The quality of the electricity in consumers' family is closely related to the situation of the distribution net work. However, the probability that network occur a single-phase-to-ground fault is extraordinary high. The line voltage among three phases remain unchanged, and the breakdown current is small, the circuit-breaker does not cut the circuit, and the system can keep working for a period after the fault happened. But the rise of the phase voltage and the arc at accident point will cause the electrical short-circuit and the destroy if the equipment. So it is valuable to discover a efficient equipment to distinguish the breakdown signal.

Write the formula in Matlab, calculate the transverse component $\mathrm{Bx}$ and the longitudinal component By of the intensity of the magnetization of the point which is underneath two detective point before and after the accident point for 3 meters. Compare 4 wave patterns intensity of themagnetization, we can get the influences that cutting-out breakdown gives to the magnetic field at different point.

\section{Acknowledgments}

The content of this thesis is supported by the college students innovations special project(Serial Number: 201510054115)

\section{Reference}

[1]. Sun B, Xue Y, Sun T, et al. A Fault Current Measurement Method for Single Phase Grounding of Non-solidly Earthed Overhead Lines[J]. Automation of Electric Power Systems, 2008.

[2].Li C, Tayjasanant T, Xu W, et al. Method for voltage-sag-source detection by investigating slope of the system trajectory[J]. IET Proceedings - Generation Transmission and Distribution, 2003, 150(3):367-372. 
[3].Sun B, Zhang C H, Sun T J, et al. Earth fault location based on transient phase current in non-solidly earthed network[J]. Power System Protection \& Control, 2012.

[4].Zhenkun L I, Chen X, Liu H, et al. Online Assessment of Distribution Network Loading Capability[J]. Automation of Electric Power Systems, 2009, 33(6):36-38. 